\title{
Lack of High-Level Resistance Mutations in HIV Type 1 BF Recombinant Strains Circulating in Northeast Brazil
}

\author{
Joana Paixao Monteiro-Cunha, Adriano Fernando Araujo,, Edson Santos, ${ }^{2}$ \\ Bernardo Galvao-Castro, ${ }^{1,2}$ and Luiz Carlos Junior Alcantara ${ }^{1,2,3}$
}

\begin{abstract}
The genetic variability and the prevalence of drug resistance-associated mutations (DRAM) of HIV-1 isolates from 50 women and 8 children from Feira de Santana, Bahia, Brazil were investigated. DNA samples were obtained and pol sequences were generated by PCR and direct sequencing. Phylogenetic analysis showed that 39 $(67.2 \%)$ samples were subtype B, four $(6.9 \%) \mathrm{F}$, one $(1.7 \%) \mathrm{C}$, and $14(24.1 \%) \mathrm{BF}$ recombinants. Four different BF recombination patterns were detected. Twelve $(20.7 \%)$ samples shared the same breakpoint within the reverse transcriptase (RT) sequence. Fifty-five $(94.8 \%)$ isolates showed several resistance-associated mutations in the RT and the protease (PR) genes. Ten (17.2\%) isolates presented mutations associated with a high level of resistance: nine $(15.5 \%)$ to nucleoside RT inhibitors (NRTI), four (6.9\%) to nonnucleoside RT inhibitors (NNRTI), and three (5.2\%) to PR inhibitors (PIs). Subtype B-infected patients had, on average, 0.5 high-level DRAM per sequence while no mutations were observed in BF recombinants, although the two groups were under ARV for a similar period of time. Our data indicate the predominance of the subtype B, followed by BF recombinants in this population, and the dissemination of a recombinant strain in Bahia, which could be related to adaptive advantages of these variants over the predominant subtype B.
\end{abstract}

\section{Introduction}

I T IS ESTIMATED тHAт 33.2 million people are living with the human immunodeficiency virus-1 (HIV-1) worldwide, of which 15.4 million are women and 2.5 million are children under 13 years. Every year, approximately 2.5 million people are infected with HIV, $18 \%$ of whom are children. ${ }^{1}$ The majority of HIV infections result from heterosexual transmission, while mother-to-child transmission (MTCT) represents the main form of HIV infection among children. MTCT can occur during pregnancy, labor, delivery, or breastfeeding and is related to multiple factors. This includes viral, maternal, behavioral, obstetric, and newborn factors. ${ }^{2}$ In Brazil, more than 544,000 AIDS cases have been documented, of which nearly 12,500 cases were associated with MTCT. ${ }^{3}$

The HIV is highly diverse and has been classified into two main types: HIV-1 and HIV-2. The HIV-1 is largely disseminated throughout the globe and is divided into three groups: $\mathrm{M}, \mathrm{O}$, and $\mathrm{N}$. The group $\mathrm{M}$ is further divided into nine subtypes (A, B, C, D, F, G, H, J, and $\mathrm{K}$ ), 43 circulating recombinant forms (CRF), and several unique recombinant forms (URF). ${ }^{4-7}$ These various genotypes present differential geographic distribution worldwide. The subtype B is more disperse while the subtype $C$ is more prevalent. ${ }^{8}$ In Brazil, subtype $B$ is the predominant genotype followed by B/F recombinants and subtypes $F$ and $C .{ }^{7,9-12}$ However, due to the vast territory and to an intense social and cultural heterogeneity, the Brazilian geographic regions have been characterized by striking differences concerning subtype distribution.

The extreme variability of HIV-1 is related to the high error rate of the reverse transcriptase enzyme and to the occurrence of homologous recombination of the viral RNA during replication. ${ }^{13}$ The accumulation of mutations favors the emergence of virus mutants that escape the immune response and ARV therapy. In Brazil, HIV-infected patients have been provided with full and free access to all Brazilian Health Surveillance Agency (Anvisa)-approved ARVs since 1996. Although it has contributed to an increase in survival and quality of life, the sequential use of ARVs has also contributed to the emergence of viral resistance, which has consequences

\footnotetext{
${ }^{1}$ Laboratório Avançado de Saúde Pública (LASP), Centro de Pesquisa Gonçalo Moniz (CPqGM), Fundação Oswaldo Cruz (FIOCRUZ), Salvador, Bahia, Brazil.

${ }^{2}$ Fundação Bahiana para o Desenvolvimento das Ciências (FBDC), Escola Bahiana de Medicina e Saúde Pública (EBMSP), Salvador, Bahia, Brazil.

${ }^{3}$ Vaccine Branch, National Cancer Institute, National Institutes of Health, Bethesda, Maryland.
} 
at the individual level, such as virological failure, and at the community level, such as secondary resistance ${ }^{14,15}$ and transmission of antiretroviral-resistant HIV, ${ }^{16}$ including MTCT.

We conducted this study in order to characterize the prevalence of DRAM and the molecular diversity of HIV isolates in a population composed by women and children from Feira de Santana, an inner city of Bahia State, in the northeast of Brazil. Our results indicate the predominance of subtype B in this population. Mutations associated with a high level of drug resistance were not detected among individuals infected with BF recombinant viruses.

\section{Materials and Methods}

\section{Population}

This study was performed with a convenience sample of 58 HIV-1-infected individuals (50 women and 8 children) followed at the Reference Center for Sexual Transmitted Disease (STD) / AIDS of Feira de Santana and recruited during gynecological follow-up (women). During 2007, blood samples were collected and sent to the Advanced Laboratory of Public Health (LASP)/CPqGM/FIOCRUZ in Bahia, Brazil for processing. All participating patients gave written informed consent. Children (2-11 years), infected through MTCT, were included with the consent of parents or guardians. Clinical and epidemiological data were obtained from medical records.

\section{DNA extraction and PCR amplification}

Genomic DNA was extracted using the Qiagen extraction kit (QIAGEN, Valencia, CA). The amplification of the pol fragment (positions 2344-3160 relative to HXB2 genome) was obtained through nested polymerase chain reaction (PCR) in a Perkin Elmer 9600 Thermal Cycler (Waltham, MA) using $500 \mathrm{ng}$ of DNA. DP10 and LR54 and DP16 and RT12 were used as outer and inner primers, respectively. The PCR cycling conditions were as follows: three cycles of denaturing at $95^{\circ} \mathrm{C}$ for $3 \mathrm{~min}$, annealing at $55^{\circ} \mathrm{C}$ for $1 \mathrm{~min}$, and primer extension at $72^{\circ} \mathrm{C}$ for $1 \mathrm{~min}$; another 35 cycles of denaturing at $95^{\circ} \mathrm{C}$ for $1 \mathrm{~min}$, annealing at $55^{\circ} \mathrm{C}$ for $45 \mathrm{~s}$, and primer extension at $72^{\circ} \mathrm{C}$ for $1 \mathrm{~min}$; and a final extension at $72^{\circ} \mathrm{C}$ for $10 \mathrm{~min}$.
The gag p17 (1150 bp) nested PCR protocol was carried out as described previously. ${ }^{17}$

\section{Sequencing and phylogenetic analysis}

PCR products were purified with Qiagen columns (QIAGEN, Valencia, CA) and sequenced in an ABI 3100 Genetic Analyzer (Applied Biosystems, Foster City, CA) using a Big Dye Terminator kit (Applied Biosystems, Foster City, $\mathrm{CA})$, the same PCR inner primers, and additional primers (LR49 and LR51). ${ }^{18}$ Sequences were assembled using SeqScape v2.1.1 software (Applied Biosystems, Foster City, CA) and checked for contamination by BLAST search against the HIV-1 sequences database (http://blast.ncbi.nlm.nih.gov).

Sequences were aligned with a subtype reference set from the Los Alamos database (http://hiv-4web.lanl.gov) and with other sequences previously characterized from Brazil and from other regions using the MUSCLE software ${ }^{19}$ and edited manually in GENEDOC. ${ }^{20}$ Phylogenetic analyses were performed using the PAUP* $4.0 \mathrm{~b} 10$ software $^{21}$ to generate neighbor-joining (NJ) and maximum likelihood (ML) trees with the General Time Reversible (GTR) model of nucleotide substitution. ${ }^{22}$ The reliability of the nodes was assessed by bootstrap analysis (1000 replicates). Furthermore, the likelihood ratio test method was used to calculate statistical support for the branches. The trees were drawn with the TreeView program. ${ }^{23}$ Recombination was identified by the bootscanning method implemented in the SimPlot software ${ }^{24}$ and the GENEDOC software was used to determine the specific crossover point by visual inspection of the alignment. Each part of the mosaic structure was confirmed by the reconstruction of bootstrapped NJ trees as described above. Recombination analyses were confirmed through the SQUEAL recombination tool. ${ }^{25}$ The $\mathrm{BF}$ recombinant forms determined by SimPlot were aligned with reference sequences of CRFs from the Los Alamos database and analyzed by phylogenetic reconstruction as described above.

\section{Drug resistance analysis}

The level of susceptibility to antiretroviral drugs was inferred by the Stanford University HIV Drug Resistance

Table 1. Clinical and laboratory Data of Individuals followed at the Reference Center for Sexual Transmitted Disease (STD)/AIDS of Feira de Santana, Brazil

\begin{tabular}{|c|c|c|c|c|c|c|c|c|}
\hline & \multirow[b]{2}{*}{ Group } & \multirow[b]{2}{*}{$\mathrm{N}$} & \multirow[b]{2}{*}{ Mean } & \multirow{2}{*}{$\begin{array}{l}\text { Standard } \\
\text { deviation }\end{array}$} & \multirow{2}{*}{$\begin{array}{l}\text { Standard } \\
\text { error mean }\end{array}$} & \multirow[b]{2}{*}{$\mathrm{p}^{\mathrm{a}}$} & \multicolumn{2}{|c|}{$95 \%$ confidence interval of the difference } \\
\hline & & & & & & & Upper & Lower \\
\hline \multirow[t]{2}{*}{ Viral load (copies/ml) } & Children & 8 & $113,251.2$ & $171,321.4$ & $60,571.2$ & 0.022 & $12,080.5$ & $152,645.2$ \\
\hline & Women & 50 & $30,888.4$ & $74,221.0$ & $10,496.4$ & & & \\
\hline \multirow[t]{2}{*}{ CD4 (cells/ml) } & Children & 5 & 922.4 & 733.2 & 327.9 & 0.012 & 110.8 & 831.8 \\
\hline & Women & 43 & 451.1 & 325.8 & 49.7 & & & \\
\hline \multirow[t]{2}{*}{$\begin{array}{l}\text { Time since diagnosis } \\
\text { (years) }\end{array}$} & Children & 8 & 5.4 & 3.5 & 1.2 & 0.171 & -0.6 & 3.4 \\
\hline & Women & 50 & 4.1 & 2.4 & 0.3 & & & \\
\hline \multirow{2}{*}{$\begin{array}{l}\text { Duration of ARV treatment } \\
\quad \text { (years) }^{\mathrm{C}}\end{array}$} & Children & 5 & 5.2 & 3.3 & 1.2 & 0.608 & -1.4 & 2.4 \\
\hline & Women & 43 & 3,3 & 2.3 & 0.3 & & & \\
\hline
\end{tabular}

${ }^{a} t$-test for equality of means.

${ }^{b}$ Only patients who had available data were considered on the mean calculation.

${ }^{c}$ Therapy-naïve patients were not included in the analysis. 
Database HIVdb program, version 6.0.5 (http://hivdb .stanford.edu), correlated with the treatment that each patient was undergoing.

\section{Statistical analysis}

Epidemiological, clinical, and laboratory (TCD4 cells and viral load) data were analyzed in the SPSS software, version 14.0 for Windows (Chicago: SPSS Inc.).

\section{Results}

\section{General characteristics of the study population}

The studied sample consisted of 50 women and eight children. The ages ranged from 20 to 73 years among women (mean $=37.9$ years) and from 2 to 11 among children (mean age $=7$ years). Of the children, five $(62.5 \%)$ were male and three $(37.5 \%)$ were female. Regarding marital status, the women were either single $(28.0 \%)$, married, and/or in a stable relationship $(56 \%)$ or a widow $(16.0 \%)$. Most of them were within the lowincome class $(83.0 \%)$ and had less than 4 years of education $(65.0 \%)$. Also, the majority of the infections were related to sexual transmission $(96.6 \%)$, while one patient was an intravenous drug user (IDU) $(1.7 \%)$ and one reported a blood transfusion $(1.7 \%)$. All the children were infected through vertical transmission during pregnancy or childbirth. Four patients were mother-child pairs (BR88FSM131-BR131FSC88, BR91FSM83-BR83FSC91, BR100FSM145-BR145FSC100, and BR110FSM111-BR111FSC110). Clinical data such as viral load, CD4 T cell counting, time elapsed since diagnosis, and time of ARV treatment are summarized in Table 1. According to the CDC Classification System (CDC, 1993), 13 (22.4\%) patients were in category A, $39(67.2 \%)$ were in category B, and six $(10.3 \%)$ were in category C. Among women, 10 (20.0\%) were in category A, $34(68.0 \%)$ were in category B, and six $(12.0 \%)$ were in category $\mathrm{C}$. Three $(37.5 \%)$ children were in category A and five $(62.5 \%)$ were in category B. Forty-eight $(82.8 \%)$ patients were under ARV therapy, while 10 (17.2\%) were therapy naive.

The phylogenetic analysis of the pol sequences revealed that $39(67.2 \%)$ isolates were subtype B, four $(6.9 \%)$ were subtype F1, and one (1.7\%) was subtype C (Fig. 1). Fourteen $(24.1 \%)$ samples did not cluster inside of any of the pure-subtype clusters. Conversely, 12 (20.7\%) of these sequences formed a monophyletic cluster in the three located between subtypes $\mathrm{B}$ and $\mathrm{F}$ (bootstrap $=70$ ). Therefore, these 14 samples were further submitted to recombination analysis through the bootscanning tool, using SimPlot software. ${ }^{24}$ The 12 sequences inside the monophyletic cluster presented the same recombination pattern between subtypes $\mathrm{F}$ and $\mathrm{B}$, while the

FIG. 1. NJ tree based on pol sequences showing phylogenetic relations between HIV-1 samples from Feira de Santana and the group $M$ reference sequences from the Los Alamos database. A group $\mathrm{O}$ sequence was used as outgroup. GTR + I+ G was the nucleotide substitution model used. Branches supported by the ML method are indicated as ${ }^{*} p<0.05$ and ${ }^{* *} p<0.001$. Bootstrap values for 1000 replicates are indicated in percent. HIV-1 sequences generated in the present study are indicated in bold. 12 sequences formed a monophyletic group outside the subtype F1 cluster (shown within the box).

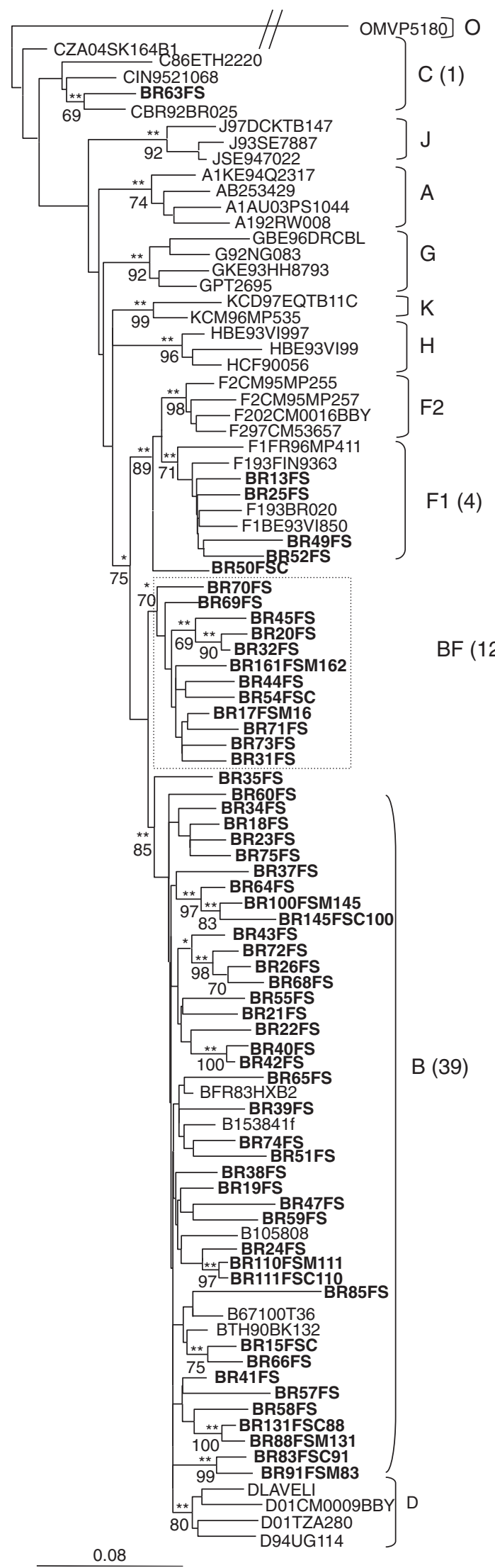


other two samples (BR35FS and BR50FS) each presented a unique recombination pattern (Fig. 2a). The crossover points of the $\mathrm{B} / \mathrm{F}$ recombinant sequences were investigated by visual inspection of the dataset. The 12 samples with the same BF pattern in the bootscanning plot also shared a breakpoint at the same location (between positions 2553 and 2584 relative to the HXB2 genome). Sample BR50FS showed a F/B/F profile within the pol fragment, with two different breakpoints: the first located between positions 2457 and 2516 and the second located between positions 2645 and 2661. The sample BR35FS presented a breakpoint at position 2460-2462. NJ and ML analyses of the individual fragments confirmed the bootscanning recombination results (Fig. 2b).

To further characterize the recombinant viruses circulating in this population, we compared their genetic pattern with recombinant sequences previously characterized in Brazil as well as with the established CRFs and pure B and F sequences. The NJ (Fig. 3) analysis shows that 12 samples with the same BF recombination pattern also formed a monophyletic cluster with previously characterized CRF28/CRF29 sequences from southeast Brazil. Sample BR50FS clustered as an outgroup of the CRF40 cluster ${ }^{26}$ and sample BR35FS did not cluster with any characterized CRF or pure subtype sequence. To investigate if the recombinants from Feira de Santana are related to a group of BF recombinants previously characterized from Salvador, the capital of Bahia State, we have compared their gag region (Fig. 3). Seven out of the 12 recombinants from Feira de Santana sharing the same breakpoint in pol amplified in the gag PCR and formed a monophyletic cluster with the recombinants from Salvador and with CRF28/ CRF29 sequences (Fig. 3). These seven viruses presented the same recombination pattern with the breakpoint located between positions 1322 and 1397. In both gag and pol analyses, two subclusters were observed inside the major monophyletic cluster: one formed by all CRF28 and CRF29 sequences plus a few BF sequences from Bahia and the other formed exclusively by BF sequences from Bahia. Sequence BR50FS was classified as subtype B in the gag fragment.

The prevalence of DRAM was investigated in the pol sequences of the HIV isolates circulating in Feira de Santana. We found several resistance-associated mutations in the reverse transcriptase (RT) and the protease (PR) regions in $55(94.8 \%)$ isolates. Ten samples (17.2\%) presented mutations associated with a high level of resistance to HIV drugs: nine (15.5\%) to NRTI, four (6.9\%) to NNRTI, and three (5.2\%) to PIs (Table 2). The mutations related to resistance to RT inhibitors were M184V (5), M41L (6), G190A (2), G190S (1), K103N (2), K101E (1), and Y181C (1); these mutations confer resistance to most ARVs. Mutations within the PR region related to PIs resistance were M46L (nelfinavir), I50L (atazanavir/r), I54L (fosamprenavir/r), V82A (indinavir/r, nelfinavir, lopinavir), N88D (nelfinavir), and N88T (nelfinavir). Among the patients who had high resistance mutations, nine were using the treatment regimen that they were resistant to, while one patient (BR100FSM145) presented a mutation associated with a drug that was not part of her antiretroviral combination. Nine (90\%) patients presented DRAM to at least one of the drugs of their ARV regimen, two (20\%) had DRAM to two of the drugs of their ARV regimen, and two $(20 \%)$ had DRAM to three of the drugs of their ARV regimen (Table 2). None of the naive patients presented mutations associated with high-level resistance. However, one naive patient (BR22FS) presented a

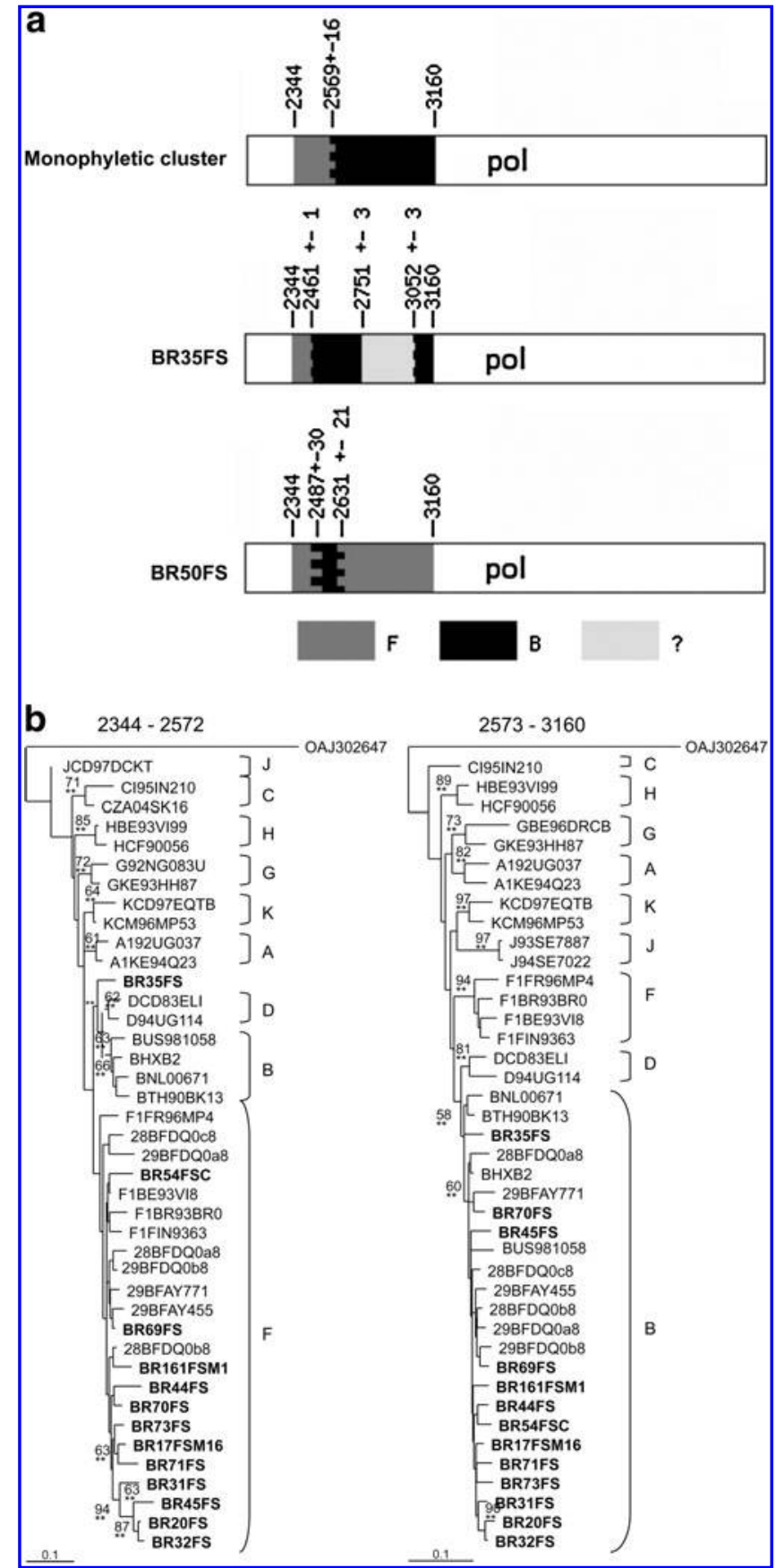

FIG. 2. (a) Patterns of recombination between subtypes B and $\mathrm{F}$ inside the pol gene found among HIV samples from Northeast Brazil. (b) Phylogenetic trees confirming the BF recombination in 10 samples with the same breakpoint: sequences were fragmented at the breakpoint and the segments were submitted to NJ analysis with the Los Alamos reference set using the GTR $+\mathrm{G}$ and the GTR $+\mathrm{I}+\mathrm{G}$ substitution models, respectively. Bootstrap values for 1000 replicates are indicated. Branches highly supported by the ML method are indicated as ${ }^{* *} p<0.001$. HIV-1 sequences generated in the present study are indicated in bold. The same method was used to confirm recombination in the other three patterns. 

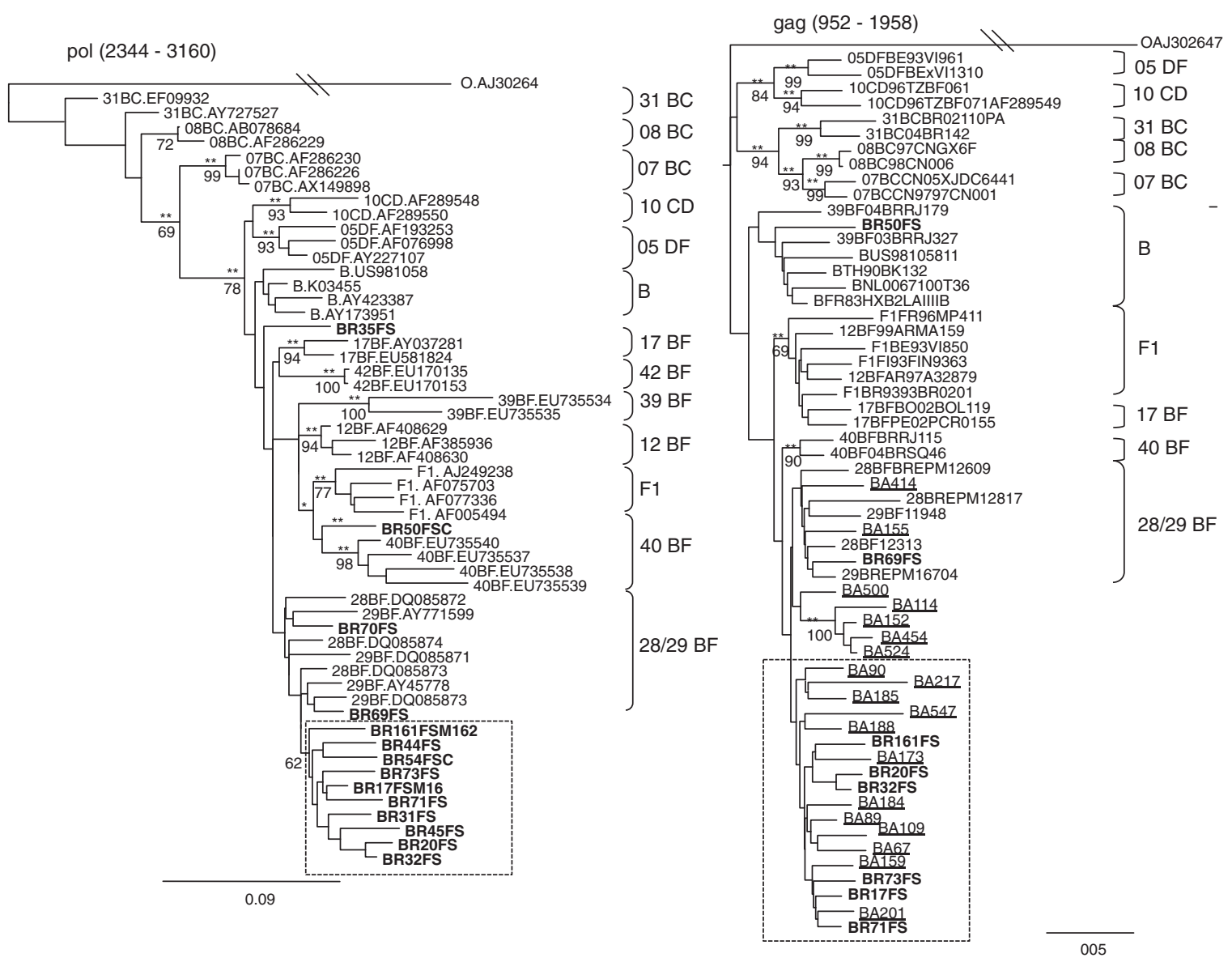

FIG. 3. Phylogenetic relationships among HIV BF recombinants from Feira de Santana, compared to other subtype B, F, and CRFs sequences based on pol and gag fragments. A group $\mathrm{O}$ sequence was used as the outgroup. Trees were constructed based on the NJ method using the GTR + I + G substitution model. Branches supported by the ML method are indicated as ${ }^{*} p<0.05$ and highly supported by ${ }^{* *} p<0.001$. Bootstrap values for 1000 replicates are indicated in percent. Recombinant sequences from Feira de Santana are indicated in bold and those from Salvador are underlined. Ten sequences formed a unique monophyletic group shown within the box.

substitution $(\mathrm{T} 69 \mathrm{~N})$ associated with low-level resistance to two NRTIs (D4T and DI). The mean number of mutations associated with a high level of resistance per virus was 0.4 among women and 0.5 among children. We have compared the clinical, epidemiological, and genetic features between subtype $B$ and BF-infected individuals undergoing ARV treatment (Table 3). Among subtype B-infected individuals, $33(84.6 \%)$ were under ARV therapy and six (15.4\%) were drug naive, while among BF infected individuals, $11(78.6 \%)$ were under ARV therapy and three (21.4\%) were drug naive. Since the mother-child pairs are linked cases, we have excluded the four children whose mothers were already included in the following analyses (one child was also drug naive). There were no significant differences between the groups regarding age, viral load, TCD4 cell count, time under ARV therapy, and time of the first HIV-positive diagnostic. All drug-naive patients were in clinical category A of the CDC classification. Among subtype B-treated individuals, two $(6.7 \%)$ were in clinical category A of the CDC classification, 23 $(76.7 \%)$ were in category $\mathrm{B}$, and five $(16.7 \%)$ were in category C. Among BF-treated patients, one $(9.1 \%)$ were in category A, nine $(81.8 \%)$ were in category $\mathrm{B}$, and one $(9.1 \%)$ was in cate- gory C. Subtype B-infected patients had, on average, 0.5 highlevel DRAM per sequence while no mutations were observed in BF recombinants $(p=0.016)$ (Table 3$)$.

\section{Discussion}

In Brazil, following a worldwide trend, the beginning of the AIDS epidemic was restricted to large urban centers, affecting specific risk groups such as homosexuals, IDUs, and hemophiliacs. However, within the past two decades, the epidemic has reached new trends, spreading to cities of medium and small size, a phenomenon described as interiorization, ${ }^{27}$ although it remains largely concentrated in urban centers. ${ }^{28}$ In this context, this work represents an effort to characterize the molecular epidemiology and the drug resistance profile of the HIV-1 circulating among women and children in Feira de Santana, an inner city in the northeast of Brazil, the region with the highest level of poverty in the country.

Feira de Santana is located $107 \mathrm{~km}$ from Salvador, the capital of Bahia State, and is the second most populous city in the state with nearly 600,000 inhabitants. It is also situated at one of the major road junctions of the northeast of the country, 


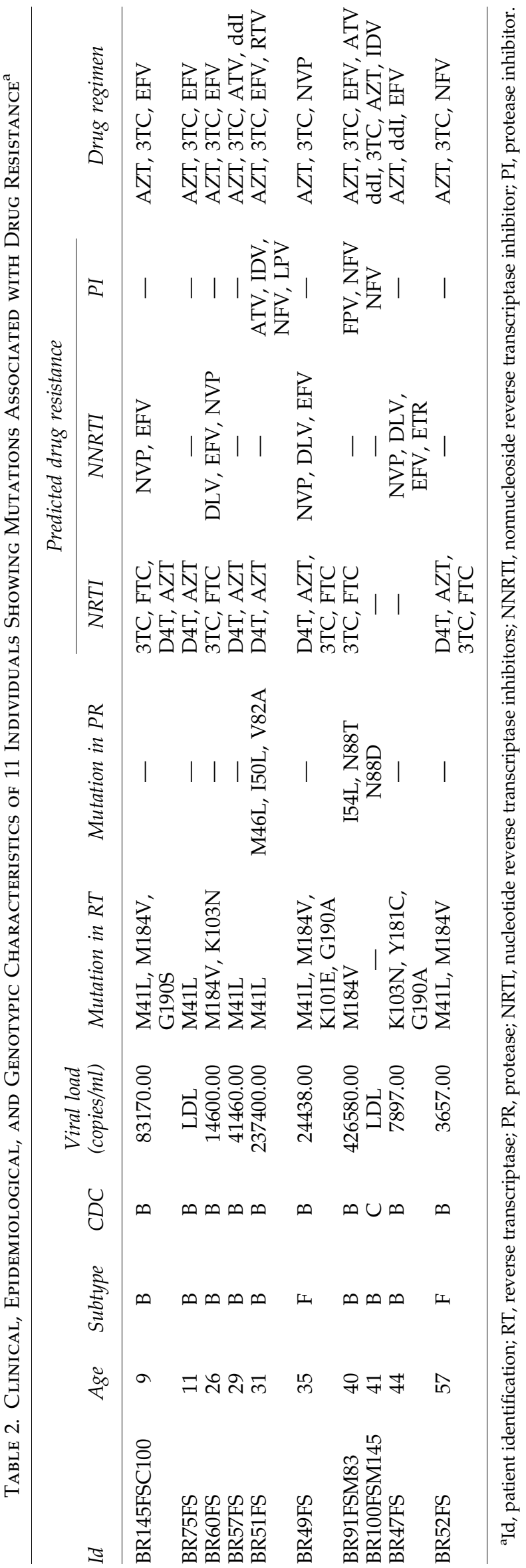

connecting it with the midwest, the south, and the rest of the northeast regions. Until March 2007, the Reference Center for STD/AIDS in Feira de Santana has reported 781 cases of HIV infections: 569 (72.9\%) men, $180(23.0 \%)$ women (male/female ratio $=3.2: 1)$, and $32(4.1 \%)$ children infected through vertical transmission.

We have studied the clinical, epidemiological, and virological characteristics of $58 \mathrm{HIV}$-infected individuals, including 50 women and eight children. Similar to what has been observed in other parts of the country, the majority was in the low-income class $(83.0 \%)$ and reported a low level of education $(65.0 \%)$. Sexual transmission was by far the predominant route of contamination. Similar data were previously observed in Salvador. ${ }^{29,30}$ We compared the viral load, T CD4 cell count, time elapsed since diagnosis (in years), and time of ARV treatment (in years) between women and children (Table 1). Although the children means were higher for all categories, there were no statistical differences between the two groups and we believe that these higher numbers are related, in part, to the small number of the group. Moreover, children usually have higher CD4 counts and baseline viral loads than adults, ${ }^{31,32}$ which may reflect the lower efficiency of an immature immune system in containing viral replication. None of the children was in the CDC clinical category C, which is characterized by AIDS-indicative conditions. A higher proportion of children (37.5\%) was in category $\mathrm{A}$, the asymptomatic stage, compared with women (20.0\%). However, a higher proportion of women was in clinical categories B $(68.0 \%)$ and C $(12.0 \%)$ compared with children $(62.5 \%$ and $0 \%$, respectively).

The phylogenetic analyses of the pol sequences showed the presence of three distinct subtypes (B, C, and F) and three different patterns of $\mathrm{BF}$ recombinant forms in this population. The subtype B was the most prevalent $(67.2 \%)$ followed by BF recombinants, which accounted for $24.1 \%$ of the viruses, F1 $(6.9 \%)$, and C $(1.7 \%)$. These data show an increased prevalence of BF recombinants if compared with previous studies in Bahia and Brazil. In 1999, Couto-Fernandez et al. reported $3.4 \%$ of $\mathrm{BF}$ recombinants among a group of individuals infected through sexual contact in Salvador. ${ }^{33}$ In 2003, Brindeiro et al. studied samples from northeast Brazil and showed 80\% $\mathrm{B}, 10 \% \mathrm{BF}, 6 \% \mathrm{~F} 1$, and $4 \% \mathrm{C} .{ }^{34} \mathrm{~A}$ recent report from our group has shown $84.0 \% \mathrm{~B}, 13.1 \% \mathrm{BF}, 2.3 \% \mathrm{~F} 1$, and $0.6 \% \mathrm{~F} / \mathrm{D}^{11}$ and another study conducted in Salvador showed, in 2007, 82.3\% of $\mathrm{B}, 13 \%$ of $\mathrm{BF}, 3.5 \%$ of $\mathrm{F}$, and $1.1 \%$ of $\mathrm{C} .{ }^{35}$ Therefore, the present work suggests that an expansion of $\mathrm{BF}$ recombinant forms in spite of the pure subtype $\mathrm{F}^{11,36,37}$ and a decrease of the prevalence of subtype B may be occurring in this region. The factors involved in the observed changes on subtype prevalence are still not understood but might involve the acquisition of genetic features that confer an increased viral fitness. In fact, the overtaking of an HIV epidemic by one genetic strain over another has been described in other regions. For example, in south Brazil and in Thailand, subtype $\mathrm{C}^{38}$ and the CRF01_AE, ${ }^{39,40}$ respectively, overtook subtype $\mathrm{B}$ and became the predominant genotypes. These observations reflect the complexity of the dynamics and evolution of HIV.

Among our samples, eight comprised four mother-child pairs (BR88FSM131-BR131FSC88, BR91FSM83-BR83FSC91, BR100FSM145-BR145FSC100, and BR110FSM111-BR111FSC110). As expected, the sequences of each pair grouped together in 
Table 3. Comparison of Clinical, Epidemiological, and Genetic Features between Subtype B AND BF-INFECTED INDIVIDUALS UNDER ARV TREATMENT

\begin{tabular}{|c|c|c|c|c|c|c|}
\hline & Group & $\mathrm{N}$ & Mean & Standard deviation & Standard error mean & $\mathrm{p}^{\mathrm{a}}$ \\
\hline \multirow[t]{2}{*}{ Age } & B & 30 & 37.20 & 10.981 & 2.005 & 0.665 \\
\hline & $\mathrm{BF}$ & 11 & 35.45 & 12.405 & 3.740 & - \\
\hline \multirow[t]{2}{*}{ Viral load (copies/ml) } & B & 30 & $37,275.93$ & $86,517.970$ & $15,795.948$ & 0.908 \\
\hline & $\mathrm{BF}$ & 11 & $33,904.50$ & $67,431.396$ & $20,331.331$ & \\
\hline \multirow[t]{2}{*}{ CD4 (cells $/ \mathrm{ml})^{\mathrm{b}}$} & B & 25 & 386.28 & 264.239 & 52.848 & 0.894 \\
\hline & $\mathrm{BF}$ & 9 & 373.67 & 161.279 & 53.760 & \\
\hline \multirow[t]{2}{*}{ Duration of ARV treatment (years) } & B & 30 & 3.37 & 2.456 & 0.448 & 0.739 \\
\hline & $\mathrm{BF}$ & 11 & 3.09 & 1.921 & 0.579 & \\
\hline \multirow[t]{2}{*}{ Time since diagnosis (years) } & $\mathrm{B}$ & 30 & 4.70 & 2.731 & 0.499 & 0.415 \\
\hline & $\mathrm{BF}$ & 11 & 3.91 & 2.700 & 0.814 & \\
\hline \multirow[t]{2}{*}{ DRAM per sample } & B & 30 & 0.50 & 1.075 & 0.196 & 0.016 \\
\hline & $\mathrm{BF}$ & 11 & 0.00 & 0.000 & 0.000 & \\
\hline
\end{tabular}

${ }^{a} t$-test for equality of means.

${ }^{b}$ Only patients who had available data were considered on the mean calculation.

both NJ and ML trees (Fig. 1) with high bootstrap levels (varying from 83 to 100 ).

Three different recombination patterns between subtypes B and $\mathrm{F}$ were observed among our samples (Fig. 2). The first pattern was represented by 12 samples that formed a monophyletic cluster (Fig. 3) and shared the same breakpoint interval in both pol and gag analyses. These sequences are most likely CRF28/CRF29 representatives as they presented a similar genetic structure in both genes. The fact that $10(\mathrm{pol})$ and $18(\mathrm{~g} a g)$ BF sequences from Bahia form a monophyletic cluster separated from CRF28/CRF29 sequences from southeast Brazil is probably the result of founder effects, but most likely traces back to the same original recombination event. Moreover, the phylogenetic analysis showed that the BF recombinants from Feira de Santana isolated in 2007 and the recombinants from Salvador isolated in $2002^{11}$ share a common ancestral pattern and present the same recombination pattern, indicating that this recombinant form is fairly disseminated in Bahia state.

The second BF pattern was represented by sample BR35FS, characterized by an initial, small $\mathrm{F}$ fragment followed by a longer B fragment. However, in its B fragment, this sample presented a region $(\sim 300 \mathrm{bp})$ that could not be assigned to any known subtype or CRF (not shown). Finally, sample BR50FS represented the third BF recombination pattern and clustered close to CRF $40^{25}$ sequences in the pol but not in the gag analysis. This could be explained by the fact that this sample showed pol breakpoints located in the same regions as the ones found in CRF40 (Fig. 2a) but did not present the gag breakpoint (position 1340) identified in the CRF40 variants. The fact that BR35FS and BR50FS breakpoint regions overlap can indicate that they are either derived from a common ancester that went through subsequent recombination processes or, most likely, that they represent different recombination events, since these two sequences were not related in the phylogenetic analyses. It is worth noting that all three recombinant patterns presented breakpoints in the beginning of the RT or at the end of the PR. Likewise, various CRFs also have crossovers at this genomic region (www.hiv.lanl.gov), confirming the existence of recombination hotspots on the HIV genome. ${ }^{41}$

Forty-eight $(82.8 \%)$ patients of this cohort were taking ARVs. Although 55 (94.8\%) individuals presented mutations in the TR and PR mutations associated with drug resistance, most of these mutations do not alter the sensitivity of the viruses to the drugs. However, nine (15.5\%) sequences showed mutations associated with a high level of resistance to RT inhibitors and three (5.2\%) to PR inhibitors (Table 2). From the individuals with high-level resistance mutations, $90 \%$ were taking at least one drug they were resistant to, $20 \%$ were taking two drugs they were resistant to, and $20 \%$ were taking three drugs they were resistant to. One sample (10\%) had a mutation associated with resistance to nelfinavir, which was not in the regimen of the patient. This patient had was diagnosed with HIV 8 years before the study, has been on treatment for 7 years, and had a controlled viral load and T CD4. Therefore, this could represent a case of transmitted drug resistance (TDR). However, the treatment history could not be explored to determine if this individual had previously been treated with nelfinavir. The majority $(60 \%)$ of the individuals with drug resistance mutations had a viral load higher than 10,000 copies $/ \mathrm{ml}$, while two $(20 \%)$ had undetectable viral loads, which would be consistent with the fact that the viral fitness drops after the acquisition of resistance mutations, making the strains less efficient. ${ }^{42}$ Sample BR145FSC100, isolated from a 7-year-old child, did not present the RT mutations D67N and V118I or the PR mutations E35D, Q58E, and N88D, all present in his mother (BR100FSM145). In this case, either a less prevalent strain without the mutations was transmitted and gained fitness in the new host environment or the mutations were reverted after the transmission. However, BR145FSC100, but not his mother, had M41L, M184V, and G190S mutations, indicating acquisition after transmission. In fact, M41L confers high resistance to AZT, M184V confers high resistance to 3TC, and G190S is associated with high resistance to $\mathrm{EFV}$; these drugs were part of the child regimen (Table 2). Similarly, patient BR91FSM83 but not her child (BR83FSC91) presented high-level DRAMs. The other two pairs had wild-type viruses.

From all six individuals in CDC category C (AIDS symptoms), only one presented high-level DRAMs. The other five patients at AIDS stage were under ARV therapy; however, they did not present high-level DRAMs. This is contrary to what we would expect, since the development of AIDS symptoms is usually related to therapy failure and the emergence of DRAMs. However, the absence of detectable 
viral resistance after treatment failure may result from factors such as the presence of drug-resistant minority viral populations, nonadherence to medications, lack of current knowledge of the association of certain mutations with drug resistance, and others. ${ }^{43}$ Moreover, there are other amino acid substitutions associated with a high level of resistance to HIV drugs that are outside the analyzed fragment, as is the case of D30N, the major DRAM for nelfinavir and mutations associated with resistance to NRTIs (codons 210, 215, and 219) and to NNRTIs (codons 225, 227, 230, 236, and 238). The majority (nine) of the patients with high-level DRAMs (Table 2) were in the B category (90.0\%). The presence of high-level DRAM was not identified among BF sequences, while among the subtype $B$ group, the mean number of DRAM per patient was 0.41 , in spite of the fact that these two groups were under the selective pressure of ARV therapy for a very similar period of time (Table 3). This difference was supported by $p=0.016$. However, other factors, not evaluated in this study, such as type of drug combination, number of different salvage therapies and adherence, might have influenced the degree of selective pressure exerted by ARVs. In fact, De Sá Filho et al. showed a high level of resistance to reverse transcriptase inhibitors in both subtypes $\mathrm{B}$ and $\mathrm{F}$ and in recombinant forms $\mathrm{B} / \mathrm{F}$ in samples from therapy-naive ${ }^{44}$ and ARV-treated patients. ${ }^{45}$ We believe that further studies involving a higher number of samples might contribute to a better understand of the evolution of these recombinant strains in the local HIV epidemic.

This study suggests that the prevalence of BF recombinants is growing in Brazil, which could be related to adaptive advantages of these strains over the predominant subtype B and point to the importance of continuous surveillance of the HIV epidemic through molecular genotyping. The monitoring of molecular subtypes, resistance mutations, expansion of HIV1 , and its transmission is crucial to support public health policies and the development of new therapies and vaccines.

\section{Sequence Data}

The new sequences were reported to GenBank under the accession numbers GU121166 to GU12122.

\section{Acknowledgments}

This work was supported by grants from the Brazilian Ministry of Health STD/AIDS Program, CNPq, FBDC, and FIOCRUZ. J.P.M. acknowledges the Fundação de Amparo à Pesquisa do Estado da Bahia (FAPESB) for a postdoctoral fellowship and support. We thank Mrs. Elisabeth Deliege Vasconcelos for editing and revising this manuscript. J.P.M. and A.F.A. contributed equally to this work.

\section{Author Disclosure Statement}

No competing financial interests exist.

\section{References}

1. UNAIDS: Report on the global AIDS epidemic. Geneva, UNAIDS. 2009.

2. Martinez AMB, Hora VP, Santos AL, Mendonza-Sasi R, Groll AZ, Soarez EAJM, et al:: Determinants of HIV-1 mother-to-child transmission in southern Brazil. Ann Brazil Acad Sci 2006;78:113-121.
3. AIDS Epidemiological Bulletin Year V, N 1, 27th to 52nd epidemiological weeks, July to December 2008, 1st to 26th epidemiological weeks, January to June 2009.

4. McCutchan FE, Carr JK, Albert J, Essex M, Goudsmit J, Asjö $\mathrm{B}$, et al.: Simplified strategy for detection of recombinant HIV-1 group $\mathrm{M}$ isolates by gag/env heteroduplex mobility assay. J Virol 2000;74:363-370.

5. Casado G, Thomson MM, Sierra M, and Najera R: Identification of a novel HIV-1 circulating ADG intersubtype recombinant form (CRF19_cpx) in Cuba. J Acquir Immune Defic Syndr 2005;40:532-537.

6. Thomson MM, Casado G, Posada D, Sierra M, and Najera R: Identification of a novel HIV-1 complex circulating recombinant form (CRF18_cpx) of Central African origin in Cuba. AIDS 2005;19:1155-1163.

7. De Sa Filho DJ, Sucupira MC, Casiero MM, Sabino EC, Diaz RS, and Janini LM : Identification of two HIV type 1 circulating recombinant forms in Brazil. AIDS Res Hum Retroviruses 2006;22:1-13.

8. Hemelaar J, Gouws E, Ghys PD, and Osmanov S: Global and regional distribution of HIV-1 genetic subtypes and recombinants in 2004. AIDS 2006;20:W13-23.

9. Salemi M, de Oliveira T, Soares MA, Pybus O, Dumans AT, Vandamme AM, et al.: Different epidemic potentials of the HIV-1B and C subtypes. J Mol Evol 2005;60:598-605.

10. Monteiro JP, Ferraro GA, Oliveira T, Goldani LZ, Kashima S, Alcantara LC, et al.: Genetic and biologic characterization of HIV type 1 subtype C isolates from south Brazil. AIDS Res Hum Retroviruses 2007;23:135-143.

11. Monteiro JP, Alcantara LC, de Oliveira T, Oliveira AM, Melo MA, Brites C, et al.: Genetic variability of human immunodeficiency virus-1 in Bahia state, northeast, Brazil: High diversity of HIV genotypes. I Med Virol 2009;81:391399.

12. Taylor BS, Sobieszczyk ME, McCutchan FE, and Hammer SM: The challenge of HIV-1 subtype diversity. N Engl J Med 2008;358:1590-1602.

13. Pinto ME and Struchiner CJ: HIV-1 diversity: A tool for studying the pandemic. Cad Saude Publica 2006;22:473-84.

14. Medeiros R, Diaz RS, and Filho AC: Estimating the length of the first antiretroviral therapy regiment durability in Sao Paulo, Brazil. Braz J Infect Dis 2002,6:298-304.

15. Caseiro MM, Golegã AAC, Etzel A, and Diaz RS: Characterization of virologic failure after an initially successful 48-week course of antiretroviral therapy in HIV/AIDS outpatients treated in Santos, Brazil. Braz J Infect Dis 2010; 12(3):doi:10.1590.

16. Sucupira MC, Caseiro MM, Alves K, Tescarollo G, Janini LM, Sabino EC, et al.: High levels of primary antiretroviral resistance genotypic mutations and $\mathrm{B} / \mathrm{F}$ recombinants in Santos, Brazil. AIDS Patient Care STDS 2007,21:116-128.

17. Guimarães ML, dos Santos Moreira A, Loureiro R, GalvaoCastro B, and Morgado MG: Brazilian Network for HIV Isolation and Characterization. High frequency of recombinant genomes in HIV type 1 samples from Brazilian southeastern and southern regions. AIDS Res Hum Retroviruses 2002;18:1261-1269.

18. Maia-Teixeira SL, Bastos FI, Hacker MA, Guimarães ML, and Morgado MG: Trends in drug resistance mutations in antiretroviral-naïve intravenous drug users of Rio de Janeiro. J Med Virol 2006;78:764-769.

19. Edgar RC: MUSCLE: Multiple sequence alignment with high accuracy and high throughput. Nucleic Acids Res 2004;32: 1792-1797. 
20. Nicholas KB, Nicholas HB Jr, and Deerfield DW II: GeneDoc: Analysis and visualization of genetic variation. EMBNET News 1997;4:1-4.

21. Swofford D: PAUP 4.0: Phylogenetic analysis using parsimony (and other methods), 4.0b2a. Sinauer Associates, Inc., Sunderland, MA, 1999.

22. Posada D and Krandall KA: MODELTEST: Testing the model of DNA substitution. Bioinformatics 1998;14: 817-818.

23. Page RD: TreeView: An application to display phylogenetic trees on personal computers. Comput Appl Biosci 1996;12: 357-358.

24. Salminen MO, Carr JK, Burke DS, and McCutchan FE: Identification of breakpoints in intergenotypic recombinants of HIV type 1 by bootscanning. AIDS Res Hum Retroviruses 1995;11:1423-1425.

25. Kosakovsky Pond SL, Posada D, Stawiski E, et al.: An evolutionary model-based algorithm for accurate phylogenetic breakpoint mapping and subtype prediction in HIV-1. PLoS Comput Biol 2009;5:e1000581.

26. Guimarães ML, Eyer-Silva WA, Couto-Fernandez JC, and Morgado MG: Identification of two new CRF_BF in Rio de Janeiro State, Brazil. AIDS 2008,22:433-438.

27. Brito AM, Castilho EA, and Szwarcwald CL: AIDS e infecção pelo HIV no Brasil: uma epidemia multifacetada. Rev Soc Bras Med Trop 2001;34:207-217.

28. Grangeiro A, Escuder MM, and Castilho EA: Magnitude and trend of the AIDS epidemic in Brazilian cities, from 2002 to 2006. Rev Saude Publica 2010;44:430-440.

29. Galvão-Castro B, Couto-Fernandez JC, Mello MA, Linharesde-Carvalho MI, Castello-Branco LR, Bongertz V, et al:: A nationwide effort to systematically monitor HIV-1 diversity in Brazil: Preliminary results. Brazilian Network for the HIV-1 Isolation and Characterization. Mem Inst Oswaldo Cruz 1996;9:335-338.

30. Dourado I, Milroy CA, Mello MA, Ferraro GA, Castro-Lima Filho H, Guimarães ML, et al:: HIV-1 seroprevalence in the general population of Salvador, Bahia State, Northeast Brazil. Cad Saude Publica 2007;23:25-32.

31. Denny T, Yogev R, Gelman R, Skuza C, Oleske J, Chadwick $\mathrm{E}$, et al.: Lymphocyte subsets in healthy children during the first 5 years of life. JAMA 1992;267:1484-1488.

32. Shearer WT, Quinn TC, LaRussa P, Lew JF, Mofenson L, Almy S, et al.: Viral load and disease progression in infants infected with human immunodeficiency virus type 1 . Women and Infants Transmission Study Group. N Engl J Med 1997;336:1337-1342.

33. Couto-Fernandez JC, Morgado MG, Bongertz V, Tanuri A, Andrade T, Brites C, et al.: HIV-1 subtyping in Salvador, Bahia, Brazil: A city with African sociodemographic characteristics. J Acquir Immune Defic Syndr 1999;22:288-293.

34. Brindeiro RM, Diaz RS, Sabino EC, Morgado MG, Pires IL, Brigido L, et al.: Brazilian Network for HIV Drug Resistance Surveillance (HIV-BResNet): A survey of chronically infected individuals. AIDS 2003;17:1063-1069.
35. Brennan CA, Brites C, Bodelle P, Golden A, Hackett J Jr, Holzmayer $\mathrm{V}$, et al.: HIV-1 strains identified in Brazilian blood donors: significant prevalence of B/F1 recombinants. AIDS Res Hum Retroviruses 2007;23:1434-1441.

36. Quarleri JF, Rubio A, Carobene M, Turk G, Vignoles $M$, Harrigan RP, et al.: HIV type $1 \mathrm{BF}$ recombinant strains exhibit different pol gene mosaic patterns: Descriptive analysis from 284 patients under treatment failure. AIDS Res Hum Retroviruses 2004;20:1100-1107.

37. Barreto CC, Nishyia A, Araujo LV, Ferreira JE, Busch MP, and Sabino EC: Trends in antiretroviral drug resistance and clade distributions among HIV-1-infected blood donors in Sao Paulo, Brazil. J Acquir Immune Defic Syndr 2006;41:338-341.

38. Soares EA, Martínez AM, Souza TM, Santos AF, Da Hora V, Silveira J, et al:: HIV-1 subtype C dissemination in southern Brazil. AIDS 2005;19:S81-S86.

39. Wasi C, Herring B, Raktham S, Vanichseni S, Mastro TD, Young NL, et al.: Determination of HIV-1 subtypes in injecting drug users in Bangkok, Thailand, using peptidebinding enzyme immunoassay and heteroduplex mobility assay: evidence of increasing infection with HIV-1 subtype E. AIDS 1995;9:843-849.

40. Arroyo MA, Phanuphak N, Krasaesub S, Sirivichayakul S, Assawadarachai V, Poltavee K, et al.: HIV type 1 molecular epidemiology among high-risk clients attending the Thai Red Cross Anonymous Clinic in Bangkok, Thailand. AIDS Res Hum Retroviruses 2010;26:5-12.

41. Minin VN, Dorman KS, Fang F, and Suchard MA: Phylogenetic mapping of recombination hotspots in human immunodeficiency virus via spatially smoothed change-point processes. Genetics 2007;175:1773-1785.

42. Nijhuis $M$, Deeks $S$, and Boucher C: Implications of antiretroviral resistance on viral fitness. Curr Opin Infect Dis 2001;14:23-28.

43. Johnson VA, Brun-Vezinet F, Clotet B, Gunthard HF, Kuritzkes DR, Pillay D, et al:: Update of the drug resistance mutations in HIV-1: December 2009. Top HIV Med 2009; 17:138-145.

44. De Sa-Filho DJ, Ambar RF, Duarte NB, Matias RB, Candido $\mathrm{V}$, Gagliani LH, et al.: HIV type 1 diversity from newly diagnosed patients in Santos metropolitan area/Brazil. AIDS Res Hum Retroviruses 2009;25:925-929.

45. De Sa-Filho DJ, Soares Mda S, Candido V, Gagliani LH, Cavaliere E, Diaz RS, et al.: HIV type 1 pol gene diversity and antiretroviral drug resistance mutations in Santos, Brazil. AIDS Res Hum Retroviruses 2008;24:347-353.

Address correspondence to: Joana Paixao Monteiro-Cunha $L A S P / C P q G M / F I O C R U Z$

Rua Waldemar Falcão 121

Candeal, Salvador, Bahia 40296-710

Brazil

E-mail: jmonteiro@bahia.fiocruz.br 
